\title{
Management of Sucking Pests of Blackgram Using Seed Treatment Chemicals
}

\author{
M. Shobharani*, Sidramappa and N.M. Sunilkumar \\ Agricultural Research Station, Bidar, University of Agricultural Sciences, \\ Raichur, Karnataka, India \\ *Corresponding author
}

\begin{abstract}
A B S T R A C T
Field experiment was conducted for two consecutive seasons under rain fed

Keywords

Sucking pests, Greengram, Imidacloprid.

\section{Article Info}

Accepted: 26 October 2017 Available Online: 10 December 2017 condition to evaluate the efficacy of Imidacloprid 60 FS a new seed dressing chemical at different doses against blackgram early season sucking pests at Agricultural Research Station, Bidar, Karnataka. Among the different doses evaluated, the higher doses of Imidacloprid 60 FS, i.e., at 10 $\mathrm{ml}$ and $15 \mathrm{ml} / \mathrm{kg}$ of seeds were proved superior in protecting the crop from the early season sucking pests viz., thrips, aphids and leafhoppers up to 4045 days after sowing and recorded significantly highest grain yield. Hence, considering the cost of plant protection, Imidacloprid 60 FS @ $10 \mathrm{ml} / \mathrm{kg}$ of seeds can be used for managing the blackgram early season sucking pests up to 40-45 days after sowing.
\end{abstract}

\section{Introduction}

Black gram [Vigna mungo (L.) Hepper] is a short-duration Kharif grain legume belongs to the family Fabaceae and is a rich source of protein which is one of the essential nutrients of human. Black gram contributes $11 \%$ to the national pulse production from an area of $14 \%$. The area under black gram in India is about 3.25 million ha with production of 1.81 million tonnes and productivity of $585 \mathrm{~kg}$ $\mathrm{ha}^{-1}$. In Karnataka, black gram is cultivated in an area of $0.94 \mathrm{lakh} / \mathrm{ha}$ with 0.41 lakh tonnes production and an average productivity of 440 $\mathrm{kg} / \mathrm{ha}$. Blackgram is attacked by different species of insect pests, among them sucking insect pests (aphid, jassids, white leaf hopper and whitefly) are of the major importance
(Islam et al., 2008). These insect pests not only reduce the vigour of the plant by sucking the sap but transmit diseases and affect photosynthesis as well (Sachan et al., 1994). The annual yield loss due to the insect pests has been estimated at about 30 per cent in urd bean and mung bean.

In order to manage these sucking pests, Farmers rely on foliar application of insecticides after their occurrence or pest population crossing Economic Threshold Level in black gram. Under such situation, insecticides of a new group seed treatment chemical emerged most promising, low cost, and selective; less polluting with least 
interference in the natural equilibrium can be used for managing the sucking pests. Among them, Imidacloprid is a neonicotinoid compound belonging to the chloronicotinyl insecticide which has gut and contact activity against insects (Maienfisch et al., 2001). After seed treatment, Imidacloprid shows systemic and residual toxicity in several crop plants and interferes with transmission of stimuli or impulses in the nervous system of insect herbivores, and gives excellent control against a broad range of commercially important sucking insect pests (Zhang et al., 2011).

Seed treatment with systemic insecticide is an integral part of pest management tactics, which is comparatively less pollutant to the environment, cost effective, selective and reported to maintain natural equilibrium (Taylor et al., 2001; Nault et al., 2004). The Imidacloprid (Gaucho $70 \mathrm{WS}$ ) is a low cost, selective and less polluting compound which is found promising against sucking pests of cotton (Patil et al., 1999; Dandale et al., 2001; Vadodaria et al., 2001; Dhawan and Simwat, 2002; Patil et al., 2004). This keeps cotton seedlings free from sucking pests at least 45 days of their age (Udikeri et al., 2007).

Seed treatment is one of the highly progressive and demandable technologies in integrated pest management (IPM) for controlling various crop pests (Taylor et al., 2001; Magalhaes et al., 2009). Therefore, the present study was undertaken to know the comparative effect of Imidacloprid (Gaucho 60 FS) as seed treating agent and other foliar insecticide on the abundance of sucking pests and yield of black gram under natural field conditions.

\section{Materials and Methods}

A field experiment was conducted to test the efficacy of different doses Imidacloprid 60 FS against sucking pests i.e., aphids, thrips and leafhoppers infesting Blackgram. The experiment was carried out at Agricultural Research Station Bidar during Kharif season 2014 and 2015 in medium black soil under rainfed conditions. The experiment was laid out in Randomized block design with eight treatments replicated thrice. The plot size of each treatment was $3 \times 4 \mathrm{~m}^{2}$ with $45 \mathrm{~cm}$ X 10 $\mathrm{cm}$ spacing. The seeds of the blackgram var.DU-1 were taken in a polythene bag and required quantity of the Imidacloprid $60 \mathrm{FS}$ (@ 5, 10 and $15 \mathrm{ml} / \mathrm{kg}$ of seeds) was added to this and stirred carefully. If necessary few ml of water can be added to get uniform coating of the chemical on the seeds and dried in shade before sowing. Before sowing the treated seeds, there should be sufficient moisture in the soil. The insecticides, Imidacloprid 17.8 SL0.3 ml/Lt, Dimethoate 30 EC1.7 ml/Lt, Neem oil (1500 ppm) @ $5 \mathrm{ml} / \mathrm{Lt}$ and NSKE 5\% were used as checks for comparison. An untreated check was also maintained. All the recommended package of practices was followed to raise the crop, except plants protection measures. Where ever necessary a spray was given to control leaf eating caterpillar and pod borers. Observations on incidence of sucking pests viz., aphids, thrips and leafhoppers were made at $10,20,30$ and 40 days after complete emergence of seedlings in all treatments on randomly selected 5 plants/plot. The data was subjected for square root transformation and statistical analysis. The seed yield was recorded plot wise at the time of harvest and converted to hectare basis and subjected for statistical analysis. The mean comparisons were made by Duncan's multiple range test (DMRT). Visual observations were made for phytotoxic effects if any, expressed after germination.

\section{Results and Discussion}

Imidacloprid 60 FS rendered protection against early season sucking pests (Table 1). 
In general, the incidence of sucking pests was high during 2015-16 compared to 2014-15. The performance of seed treatment / dressers used in the present study was consistent in both the seasons.

The effectiveness of different doses of Imidacloprid 60 FS for seed treatments and foliar spray of different insecticides and plant products against management of sucking pests of blackgram under field condition during 2014-15 is presented in the Table 1. The Imidacloprid seed treatment showed significantly lower abundance of aphid population which ranged from 1.89 to 3.89 aphids per top three leaves than other foliar spray treatments which showed 11.67 to 12.33 aphids per top three leaves at 15 days after sowing. At 30 days after sowing lowest aphid population of 2.87 and 3.33 aphids / top three leaves was recorded in the plots treated with Imidacloprid 60 FS @ $15 \mathrm{ml} / \mathrm{Kg}$ of seeds and $10 \mathrm{ml} / \mathrm{kg}$ of seeds respectively.

These two treatments were followed by Imidacloprid $17.8 \mathrm{SL} @ 0.3 \mathrm{ml} / \mathrm{Lt}$ of water with 4.56 aphids/top three leaves, Dimethoate 30 EC @ $1.7 \mathrm{ml} / / \mathrm{Lt}$ of water with 4.89 aphids/top three leaves, Neem oil (1500 ppm) @ $5 \mathrm{ml} / \mathrm{Lt}$ of water with 6.00 aphids/top three leaves, seed treatment with Imidacloprid 60 FS@ $5 \mathrm{ml} / \mathrm{Kg}$ of seeds with 6.67 aphids/top three leaves and NSKE $5 \%$ with 7.11 aphids/top three leaves. The highest aphid population was noticed in untreated control with 19.00 aphids/top three leaves.

At 45 days after sowing aphid population started to build up in all the treatment which ranged from 7.67 to 13.56 aphids/ top three leaves. However, highest aphid population was recorded in untreated control with 24.11 aphids/ top three leaves. The same trend was followed at 60 days after sowing with decreasing in aphid population in all treatments.
During 2015-16 (Table 2) same trend was followed with respect the aphid population observed on top three leaves per plant in each treatment at $15 \mathrm{DAS}, 30 \mathrm{DAS}, 45 \mathrm{DAS}$ and 60 DAS.

The thrips population at 15 days after sowing was found significantly lower in the plot (Table 1) where, seed treatment with Imidacloprid 60 FS @ 15 ml/ Kg of seeds and $10 \mathrm{ml} / \mathrm{kg}$ of seeds was done by recording 1.00 and 1.22 thrips/top three leaves respectively. This was followed by Imidacloprid 60 FS @ 5 $\mathrm{ml} / \mathrm{Kg}$ of seeds with 2.33 thrips/top three leaves. Whereas the untreated plots the thrips population ranged from 4.22 to 5.00 thrips/ top three leaves. Thirty days after sowing lowest thrips population of 2.00 thrips / top three leaves was recorded in the plots where Imidacloprid 17.8 SL@0.3 ml/ Lt of water and Dimethoate 30 EC @ $1.7 \mathrm{ml} / / \mathrm{Lt}$ of water was sprayed. This was followed by treatments where seeds treated with Imidacloprid 60 FS @ $15 \mathrm{ml} / \mathrm{Kg}$ and Imidacloprid $60 \mathrm{FS} @ 10$ $\mathrm{ml} / \mathrm{kg}$ of seeds recorded 2.11 and 2.22 thrips / top three leaves respectively.

These treatments were followed by Neem oil (1500 ppm) @ 5ml/Lt of water with 3.00 thrips/top three leaves, Imidacloprid 60 FS @ $5 \mathrm{ml} / \mathrm{Kg}$ of seeds with 3.11 thrips/top three leaves, and NSKE 5\% with 4.22 thrips/top three leaves. The highest thrips population was noticed in untreated control with 8.44 thrips/top three leaves. At 45 days after sowing thrips population started to build up in all the treatment which ranged from 4.78 to 9.44 thrips/top three leaves. However, highest thrips population was recorded in untreated control with 13.78 thrips/ top three leaves. During 60 days after sowing thrips population decreased in all treatments. During 2015-16 (Table 2) same trend was followed with respect the thrips population observed on top three leaves per plant in each treatment at 15 DAS, 30 DAS, 45 DAS and 60 DAS. 
Table.1 Management of sucking pests of Blackgram during 2014-15 Kharif

\begin{tabular}{|c|c|c|c|c|c|c|c|c|c|c|c|c|c|c|}
\hline \multirow[b]{2}{*}{ Treatments } & \multirow[b]{2}{*}{ Dose } & \multicolumn{4}{|c|}{ No. of aphids/ top three leaves } & \multicolumn{4}{|c|}{ No. of thrips/ top three leaves } & \multicolumn{4}{|c|}{ No. of leafhoppers/ top three leaves } & \multirow{2}{*}{$\begin{array}{c}\text { Yield } \\
\text { (Qtl. } \\
\text { /ha) }\end{array}$} \\
\hline & & $\begin{array}{c}15 \\
\text { DAS } \\
\end{array}$ & $\begin{array}{c}30 \\
\text { DAS }\end{array}$ & $\begin{array}{c}45 \\
\text { DAS } \\
\end{array}$ & $\begin{array}{c}60 \\
\text { DAS }\end{array}$ & $\begin{array}{c}15 \\
\text { DAS } \\
\end{array}$ & $\begin{array}{c}30 \\
\text { DAS }\end{array}$ & $\begin{array}{c}45 \\
\text { DAS }\end{array}$ & $\begin{array}{c}60 \\
\text { DAS } \\
\end{array}$ & $\begin{array}{c}15 \\
\text { DAS } \\
\end{array}$ & $\begin{array}{c}30 \\
\text { DAS } \\
\end{array}$ & $\begin{array}{c}45 \\
\text { DAS } \\
\end{array}$ & $60 \mathrm{DAS}$ & \\
\hline 1. Imidacloprid $60 \mathrm{FS}$ & $\begin{array}{l}5 \mathrm{ml} / \mathrm{Kg} \\
\text { of seeds }\end{array}$ & $\begin{array}{c}3.89 \\
(2.20) \\
\end{array}$ & $\begin{array}{c}6.67 \\
(2.77) \\
\end{array}$ & $\begin{array}{l}11.00 \\
(3.46) \\
\end{array}$ & $\begin{array}{c}8.89 \\
(3.14) \\
\end{array}$ & $\begin{array}{c}2.33 \\
(1.82) \\
\end{array}$ & $\begin{array}{c}3.11 \\
(2.03) \\
\end{array}$ & $\begin{array}{c}7.67 \\
(2.94) \\
\end{array}$ & $\begin{array}{c}6.11 \\
(2.66) \\
\end{array}$ & $\begin{array}{c}1.89 \\
(1.68) \\
\end{array}$ & $\begin{array}{c}3.89 \\
(2.20) \\
\end{array}$ & $\begin{array}{c}5.22 \\
(2.49) \\
\end{array}$ & $\begin{array}{c}3.33 \\
(2.08) \\
\end{array}$ & $\begin{array}{r}4.67 \\
\text { de }\end{array}$ \\
\hline 2. Imidacloprid $60 \mathrm{FS}$ & $\begin{array}{l}10 \mathrm{ml} \\
/ \mathrm{Kg} \text { of } \\
\text { seeds } \\
\end{array}$ & $\begin{array}{c}2.11 \\
(1.75) \\
\end{array}$ & $\begin{array}{c}3.33 \\
(2.07) \\
\end{array}$ & $\begin{array}{c}8.33 \\
(3.05) \\
\end{array}$ & $\begin{array}{r}7.33 \\
(2.89) \\
\end{array}$ & $\begin{array}{c}1.22 \\
(1.49) \\
\end{array}$ & $\begin{array}{c}2.22 \\
(1.79) \\
\end{array}$ & $\begin{array}{c}5.22 \\
(2.49) \\
\end{array}$ & $\begin{array}{c}3.44 \\
(2.10) \\
\end{array}$ & $\begin{array}{c}1.00 \\
(1.41) \\
\end{array}$ & $\begin{array}{c}2.33 \\
(1.81) \\
\end{array}$ & $\begin{array}{c}4.00 \\
(2.24) \\
\end{array}$ & $\begin{array}{c}2.00 \\
(1.73) \\
\end{array}$ & $\begin{array}{r}6.20 \\
\mathrm{~b} \\
\end{array}$ \\
\hline 3. Imidacloprid $60 \mathrm{FS}$ & $\begin{array}{l}15 \mathrm{ml} \\
/ \mathrm{Kg} \text { of } \\
\text { seeds }\end{array}$ & $\begin{array}{c}1.89 \\
(1.70) \\
\end{array}$ & $\begin{array}{c}2.87 \\
(1.96) \\
\end{array}$ & $\begin{array}{c}7.67 \\
(2.94) \\
\end{array}$ & $\begin{array}{c}6.11 \\
(2.67) \\
\end{array}$ & $\begin{array}{c}1.00 \\
(1.41) \\
\end{array}$ & $\begin{array}{c}2.11 \\
(1.76) \\
\end{array}$ & $\begin{array}{c}4.78 \\
(2.40) \\
\end{array}$ & $\begin{array}{c}2.89 \\
(1.96) \\
\end{array}$ & $\begin{array}{c}0.56 \\
(1.25) \\
\end{array}$ & $\begin{array}{c}1.67 \\
(1.63) \\
\end{array}$ & $\begin{array}{c}3.11 \\
(2.03) \\
\end{array}$ & $\begin{array}{c}1.67 \\
(1.63) \\
\end{array}$ & $\begin{array}{r}7.94 \\
\mathrm{a} \\
\end{array}$ \\
\hline $\begin{array}{l}\text { 4. Imidacloprid } 17.8 \\
\text { SL }\end{array}$ & $\begin{array}{l}0.3 \mathrm{ml} / \\
\mathrm{Lt} \\
\end{array}$ & $\begin{array}{l}12.67 \\
(3.70) \\
\end{array}$ & $\begin{array}{c}4.56 \\
(2.36) \\
\end{array}$ & $\begin{array}{c}9.95 \\
(3.31) \\
\end{array}$ & $\begin{array}{r}6.00 \\
(2.63) \\
\end{array}$ & $\begin{array}{c}4.56 \\
(2.35) \\
\end{array}$ & $\begin{array}{c}2.00 \\
(1.73) \\
\end{array}$ & $\begin{array}{c}5.56 \\
(2.56) \\
\end{array}$ & $\begin{array}{l}3.78 \\
(2.18) \\
\end{array}$ & $\begin{array}{c}4.44 \\
(2.33) \\
\end{array}$ & $\begin{array}{c}3.00 \\
(1.99) \\
\end{array}$ & $\begin{array}{c}4.22 \\
(2.28) \\
\end{array}$ & $\begin{array}{c}1.67 \\
(1.63) \\
\end{array}$ & $\begin{array}{r}5.96 \\
\mathrm{bc} \\
\end{array}$ \\
\hline $\begin{array}{l}\text { 5. Neem oil (1500 } \\
\text { ppm) }\end{array}$ & $5 \mathrm{ml} / \mathrm{Lt}$ & $\begin{array}{l}12.00 \\
(3.60) \\
\end{array}$ & $\begin{array}{c}6.00 \\
(2.65) \\
\end{array}$ & $\begin{array}{l}11.11 \\
(3.47) \\
\end{array}$ & $\begin{array}{c}7.56 \\
(2.93) \\
\end{array}$ & $\begin{array}{c}4.78 \\
(2.40) \\
\end{array}$ & $\begin{array}{c}3.00 \\
(1.99) \\
\end{array}$ & $\begin{array}{c}7.22 \\
(2.86) \\
\end{array}$ & $\begin{array}{l}5.89 \\
(2.62) \\
\end{array}$ & $\begin{array}{c}3.89 \\
(2.21) \\
\end{array}$ & $\begin{array}{c}3.11 \\
(2.02) \\
\end{array}$ & $\begin{array}{c}5.56 \\
(2.56) \\
\end{array}$ & $\begin{array}{c}3.22 \\
(2.05) \\
\end{array}$ & $\begin{array}{r}4.89 \\
\text { ef } \\
\end{array}$ \\
\hline 6. NSKE & $5 \%$ & $\begin{array}{l}11.78 \\
(3.57) \\
\end{array}$ & $\begin{array}{c}7.11 \\
(2.84) \\
\end{array}$ & $\begin{array}{l}13.56 \\
(3.80) \\
\end{array}$ & $\begin{array}{c}9.44 \\
(3.23) \\
\end{array}$ & $\begin{array}{c}4.67 \\
(2.38) \\
\end{array}$ & $\begin{array}{c}4.22 \\
(2.28) \\
\end{array}$ & $\begin{array}{c}9.44 \\
(3.23) \\
\end{array}$ & $\begin{array}{l}4.67 \\
(2.38) \\
\end{array}$ & $\begin{array}{l}4.78 \\
(2.40) \\
\end{array}$ & $\begin{array}{c}4.22 \\
(2.28) \\
\end{array}$ & $\begin{array}{c}7.11 \\
(2.85) \\
\end{array}$ & $\begin{array}{c}5.33 \\
(2.52) \\
\end{array}$ & $3.32 \mathrm{f}$ \\
\hline 7. Dimethoate $30 \mathrm{EC}$ & $\begin{array}{l}1.7 \mathrm{ml} / \\
\mathrm{Lt}\end{array}$ & $\begin{array}{l}11.67 \\
(3.56) \\
\end{array}$ & $\begin{array}{c}4.89 \\
(2.40) \\
\end{array}$ & $\begin{array}{c}9.44 \\
(3.23) \\
\end{array}$ & $\begin{array}{c}6.89 \\
(2.81) \\
\end{array}$ & $\begin{array}{c}5.00 \\
(2.44) \\
\end{array}$ & $\begin{array}{c}2.00 \\
(1.72) \\
\end{array}$ & $\begin{array}{c}6.09 \\
(2.66) \\
\end{array}$ & $\begin{array}{c}4.1 \\
(2.26) \\
\end{array}$ & $\begin{array}{c}4.33 \\
(2.31) \\
\end{array}$ & $\begin{array}{c}3.11 \\
(2.03) \\
\end{array}$ & $\begin{array}{c}5.11 \\
(2.47) \\
\end{array}$ & $\begin{array}{c}2.56 \\
(1.87) \\
\end{array}$ & $\begin{array}{r}4.98 \\
\mathrm{~cd} \\
\end{array}$ \\
\hline \multirow[t]{4}{*}{ 8. Control } & - & $\begin{array}{l}12.33 \\
(3.64) \\
\end{array}$ & $\begin{array}{l}19.00 \\
(4.47) \\
\end{array}$ & $\begin{array}{l}24.11 \\
(5.00) \\
\end{array}$ & $\begin{array}{l}18.11 \\
(4.36) \\
\end{array}$ & $\begin{array}{c}4.22 \\
(2.28) \\
\end{array}$ & $\begin{array}{c}8.44 \\
(3.07) \\
\end{array}$ & $\begin{array}{l}13.78 \\
(3.83) \\
\end{array}$ & $\begin{array}{l}10.78 \\
(3.43) \\
\end{array}$ & $\begin{array}{c}4.11 \\
(2.25) \\
\end{array}$ & $\begin{array}{c}7.56 \\
(2.93) \\
\end{array}$ & $\begin{array}{l}10.00 \\
(3.28) \\
\end{array}$ & $\begin{array}{c}6.11 \\
(2.65) \\
\end{array}$ & $\begin{array}{r}2.78 \\
\mathrm{fg} \\
\end{array}$ \\
\hline & SEm \pm & 0.13 & 0.13 & 0.16 & 0.12 & 0.09 & 0.10 & 0.14 & 0.10 & 0.09 & 0.11 & 0.13 & 0.10 & 0.41 \\
\hline & $\mathrm{CD}(0.05)$ & 0.39 & 0.40 & 0.48 & 0.35 & 0.26 & 0.31 & 0.41 & 0.30 & 0.28 & 0.33 & 0.38 & 0.31 & 1.24 \\
\hline & CV (\%) & 7.45 & 8.54 & 7.78 & 6.50 & 7.19 & 8.69 & 8.22 & 7.08 & 8.16 & 9.02 & 8.60 & 8.65 & 13.69 \\
\hline
\end{tabular}

Means followed by same letter (s) are not significantly different (DMRT, $\mathrm{p} \leq 0.05$ ) 
Table.2 Management of sucking pests of Blackgram during 2015-16 Kharif

\begin{tabular}{|c|c|c|c|c|c|c|c|c|c|c|c|c|c|c|}
\hline \multirow[b]{2}{*}{ Treatments } & \multirow[b]{2}{*}{ Dose } & \multicolumn{4}{|c|}{ No. of aphids/ top three leaves } & \multicolumn{4}{|c|}{ No. of thrips/ top three leaves } & \multicolumn{4}{|c|}{$\begin{array}{c}\text { No. of leafhoppers/ top three } \\
\text { leaves }\end{array}$} & \multirow{2}{*}{$\begin{array}{l}\text { Yield } \\
\text { (Qtl. } \\
\text { /ha) }\end{array}$} \\
\hline & & \begin{tabular}{|l|}
15 \\
DAS \\
\end{tabular} & \begin{tabular}{|l|}
30 \\
DAS \\
\end{tabular} & \begin{tabular}{|l}
45 \\
DAS \\
\end{tabular} & \begin{tabular}{|l|}
60 \\
DAS \\
\end{tabular} & $\begin{array}{l}15 \\
\text { DAS } \\
\end{array}$ & \begin{tabular}{|l|}
30 \\
DAS \\
\end{tabular} & \begin{tabular}{|l}
45 \\
DAS \\
\end{tabular} & \begin{tabular}{|l|}
60 \\
DAS \\
\end{tabular} & $\begin{array}{c}15 \\
\text { DAS } \\
\end{array}$ & $\begin{array}{c}30 \\
\text { DAS } \\
\end{array}$ & $\begin{array}{c}45 \\
\text { DAS } \\
\end{array}$ & $\begin{array}{c}60 \\
\text { DAS } \\
\end{array}$ & \\
\hline $\begin{array}{l}\text { 1. Imidacloprid } 60 \\
\text { FS }\end{array}$ & $\begin{array}{l}5 \mathrm{ml} / \mathrm{Kg} \\
\text { of seeds }\end{array}$ & $\begin{array}{c}4.67 \\
(2.38) \\
\end{array}$ & $\begin{array}{r}7.89 \\
(2.98) \\
\end{array}$ & $\begin{array}{l}12.00 \\
(3.60) \\
\end{array}$ & $\begin{array}{c}9.33 \\
(3.21) \\
\end{array}$ & $\begin{array}{c}2.44 \\
(1.85) \\
\end{array}$ & $\begin{array}{c}3.22 \\
(2.05) \\
\end{array}$ & $\begin{array}{c}7.67 \\
(2.94) \\
\end{array}$ & $\begin{array}{c}9.89 \\
(3.30) \\
\end{array}$ & $\begin{array}{c}1.89 \\
(1.70) \\
\end{array}$ & $\begin{array}{c}3.11 \\
(2.03) \\
\end{array}$ & $\begin{array}{c}5.56 \\
(2.56) \\
\end{array}$ & $\begin{array}{c}3.67 \\
(2.15) \\
\end{array}$ & $5.22 \mathrm{ef}$ \\
\hline $\begin{array}{l}\text { 2. Imidacloprid } 60 \\
\text { FS }\end{array}$ & $\begin{array}{l}10 \mathrm{ml} / \mathrm{Kg} \\
\text { of seeds }\end{array}$ & $\begin{array}{c}2.89 \\
(1.97) \\
\end{array}$ & $\begin{array}{l}4.00 \\
(2.24) \\
\end{array}$ & $\begin{array}{c}9.44 \\
(3.23) \\
\end{array}$ & $\begin{array}{c}7.22 \\
(2.87) \\
\end{array}$ & $\begin{array}{c}2.56 \\
(1.89) \\
\end{array}$ & $\begin{array}{c}2.11 \\
(1.76) \\
\end{array}$ & $\begin{array}{c}3.89 \\
(2.21) \\
\end{array}$ & $\begin{array}{c}6.56 \\
(2.75) \\
\end{array}$ & $\begin{array}{c}0.67 \\
(1.29) \\
\end{array}$ & $\begin{array}{c}1.33 \\
(1.53) \\
\end{array}$ & $\begin{array}{c}3.67 \\
(2.16) \\
\end{array}$ & $\begin{array}{c}1.89 \\
(1.70) \\
\end{array}$ & $8.43 \mathrm{ab}$ \\
\hline $\begin{array}{l}\text { 3. Imidacloprid } 60 \\
\text { FS }\end{array}$ & $\begin{array}{l}15 \mathrm{ml} / \mathrm{Kg} \\
\text { of seeds }\end{array}$ & $\begin{array}{c}2.44 \\
(1.85) \\
\end{array}$ & $\begin{array}{l}3.78 \\
(2.19) \\
\end{array}$ & $\begin{array}{r}8.67 \\
(3.11) \\
\end{array}$ & $\begin{array}{c}7.00 \\
(2.83) \\
\end{array}$ & $\begin{array}{c}2.22 \\
(1.79) \\
\end{array}$ & $\begin{array}{c}1.89 \\
(1.70) \\
\end{array}$ & $\begin{array}{c}3.00 \\
(2.00) \\
\end{array}$ & $\begin{array}{c}6.33 \\
(2.71) \\
\end{array}$ & $\begin{array}{c}0.22 \\
(1.10) \\
\end{array}$ & $\begin{array}{c}1.00 \\
(1.41) \\
\end{array}$ & $\begin{array}{c}3.44 \\
(2.11) \\
\end{array}$ & $\begin{array}{c}1.78 \\
(1.67) \\
\end{array}$ & $9.42 \mathrm{a}$ \\
\hline $\begin{array}{l}\text { 4. Imidacloprid } 17.8 \\
\text { SL }\end{array}$ & $0.3 \mathrm{ml} / \mathrm{Lt}$ & \begin{tabular}{|l}
13.78 \\
$(3.84)$ \\
\end{tabular} & $\begin{array}{r}4.89 \\
(2.43) \\
\end{array}$ & $\begin{array}{c}9.78 \\
(3.28) \\
\end{array}$ & $\begin{array}{c}6.89 \\
(2.81) \\
\end{array}$ & $\begin{array}{c}4.67 \\
(2.38) \\
\end{array}$ & $\begin{array}{c}1.67 \\
(1.63) \\
\end{array}$ & $\begin{array}{c}4.78 \\
(2.40) \\
\end{array}$ & $\begin{array}{c}5.11 \\
(2.47) \\
\end{array}$ & $\begin{array}{c}4.89 \\
(2.43) \\
\end{array}$ & $\begin{array}{c}1.89 \\
(1.70) \\
\end{array}$ & $\begin{array}{c}3.22 \\
(2.05) \\
\end{array}$ & $\begin{array}{c}1.67 \\
(1.63) \\
\end{array}$ & $7.86 \mathrm{bc}$ \\
\hline $\begin{array}{l}\text { 5. Neem } \\
\text { oil(1500ppm) }\end{array}$ & $5 \mathrm{~m}$ & $\begin{array}{l}14.78 \\
(3.97) \\
\end{array}$ & $\begin{array}{l}7.33 \\
(2.89) \\
\end{array}$ & $\begin{array}{l}12.56 \\
(3.68) \\
\end{array}$ & $\begin{array}{c}8.44 \\
(3.07) \\
\end{array}$ & $\begin{array}{c}5.11 \\
(2.47) \\
\end{array}$ & $\begin{array}{c}2.78 \\
(1.94) \\
\end{array}$ & $\begin{array}{c}5.44 \\
(2.52) \\
\end{array}$ & $\begin{array}{c}6.11 \\
(2.67) \\
\end{array}$ & $\begin{array}{c}5.00 \\
(2.45) \\
\end{array}$ & $\begin{array}{c}3.00 \\
(2.00) \\
\end{array}$ & $\begin{array}{l}5.78 \\
(2.60) \\
\end{array}$ & $\begin{array}{r}3.22 \\
(2.05) \\
\end{array}$ & $5.84 \mathrm{de}$ \\
\hline 6. NSKE & $5 \%$ & $\begin{array}{l}14.00 \\
(3.87) \\
\end{array}$ & $\begin{array}{r}8.67 \\
(3.11) \\
\end{array}$ & $\begin{array}{l}15.44 \\
(4.05) \\
\end{array}$ & $\begin{array}{l}10.56 \\
(3.40) \\
\end{array}$ & $\begin{array}{c}5.44 \\
(5.57) \\
\end{array}$ & $\begin{array}{c}5.67 \\
(2.58) \\
\end{array}$ & $\begin{array}{c}7.56 \\
(2.92) \\
\end{array}$ & $\begin{array}{c}9.22 \\
(3.20) \\
\end{array}$ & $\begin{array}{c}5.44 \\
(2.54) \\
\end{array}$ & $\begin{array}{c}6.33 \\
(2.70) \\
\end{array}$ & $\begin{array}{c}6.56 \\
(2.73) \\
\end{array}$ & $\begin{array}{r}2.89 \\
(1.97) \\
\end{array}$ & $4.18 \mathrm{fg}$ \\
\hline $\begin{array}{l}\text { 7. Dimethoate } 30 \\
\text { EC }\end{array}$ & $1.7 \mathrm{ml} / \mathrm{Lt}$ & $\begin{array}{l}14.67 \\
(3.95) \\
\end{array}$ & $\begin{array}{r}6.00 \\
(2.63) \\
\end{array}$ & $\begin{array}{l}10.56 \\
(3.39) \\
\end{array}$ & $\begin{array}{c}7.56 \\
(2.91) \\
\end{array}$ & $\begin{array}{c}5.22 \\
(2.49) \\
\end{array}$ & $\begin{array}{c}3.22 \\
(1.94) \\
\end{array}$ & $\begin{array}{c}4.89 \\
(2.42) \\
\end{array}$ & $\begin{array}{c}5.33 \\
(2.49) \\
\end{array}$ & $\begin{array}{c}4.67 \\
(2.38) \\
\end{array}$ & $\begin{array}{c}2.44 \\
(1.84) \\
\end{array}$ & $\begin{array}{r}4.89 \\
(2.40) \\
\end{array}$ & $\begin{array}{c}2.56 \\
(1.87) \\
\end{array}$ & $6.88 \mathrm{~cd}$ \\
\hline \multirow[t]{4}{*}{ 8. Control } & - & $\begin{array}{l}14.11 \\
(3.86) \\
\end{array}$ & $\begin{array}{l}25.44 \\
(5.13) \\
\end{array}$ & $\begin{array}{l}28.33 \\
(5.41) \\
\end{array}$ & $\begin{array}{l}24.22 \\
(5.01) \\
\end{array}$ & $\begin{array}{c}4.56 \\
(2.33) \\
\end{array}$ & $\begin{array}{c}9.67 \\
(3.26) \\
\end{array}$ & $\begin{array}{l}15.22 \\
(4.02) \\
\end{array}$ & $\begin{array}{l}12.22 \\
(3.62) \\
\end{array}$ & $\begin{array}{c}4.78 \\
(2.38) \\
\end{array}$ & $\begin{array}{c}9.11 \\
(3.17) \\
\end{array}$ & $\begin{array}{l}11.44 \\
(3.52) \\
\end{array}$ & $\begin{array}{c}7.44 \\
(2.89) \\
\end{array}$ & $2.96 \mathrm{~h}$ \\
\hline & SEm \pm & 0.12 & 0.14 & 0.13 & 0.12 & 0.09 & 0.11 & 0.12 & 0.13 & 0.09 & 0.10 & 0.14 & 0.11 & 0.37 \\
\hline & $\mathrm{CD}(0.05)$ & 0.38 & 0.41 & 0.40 & 0.35 & 0.27 & 0.34 & 0.36 & 0.38 & 0.26 & 0.29 & 0.41 & 0.32 & 1.12 \\
\hline & CV $(\%)$ & 6.70 & 7.94 & 6.16 & 6.10 & 6.88 & 9.12 & 7.58 & 7.54 & 7.35 & 8.01 & 9.31 & 9.17 & 9.91 \\
\hline
\end{tabular}


Table.3 Management of sucking pests of Blackgram during 2014-15 and 2015-16 Kharif (Pooled)

\begin{tabular}{|c|c|c|c|c|c|c|c|c|c|c|c|c|c|c|}
\hline \multirow{2}{*}{ Treatments } & \multirow[b]{2}{*}{ Dose } & \multicolumn{4}{|c|}{ No. of aphids/ top three leaves } & \multicolumn{4}{|c|}{ No. of thrips/ top three leaves } & \multicolumn{4}{|c|}{$\begin{array}{l}\text { No. of leafhoppers/ top three } \\
\text { leaves }\end{array}$} & \multirow{2}{*}{$\begin{array}{l}\text { Yield } \\
\text { (Qtl. } \\
\text { /ha) }\end{array}$} \\
\hline & & \begin{tabular}{|l|}
15 \\
DAS \\
\end{tabular} & $\begin{array}{l}30 \\
\text { DAS } \\
\end{array}$ & \begin{tabular}{|l|}
45 \\
DAS \\
\end{tabular} & $\begin{array}{l}60 \\
\text { DAS } \\
\end{array}$ & $\begin{array}{l}15 \\
\text { DAS } \\
\end{array}$ & $\begin{array}{l}30 \\
\text { DAS } \\
\end{array}$ & $\begin{array}{l}45 \\
\text { DAS } \\
\end{array}$ & $\begin{array}{l}60 \\
\text { DAS } \\
\end{array}$ & $\begin{array}{c}15 \\
\text { DAS } \\
\end{array}$ & $\begin{array}{c}30 \\
\text { DAS }\end{array}$ & $\begin{array}{c}45 \\
\text { DAS }\end{array}$ & $\begin{array}{c}60 \\
\text { DAS }\end{array}$ & \\
\hline $\begin{array}{l}\text { 1. Imidac } \\
\text { FS }\end{array}$ & $\begin{array}{l}\mathrm{al} / \mathrm{Kg} \\
\text { seeds } \\
\end{array}$ & $\begin{array}{r}4.28 \\
(2.29) \\
\end{array}$ & $\begin{array}{c}7.28 \\
(2.87) \\
\end{array}$ & $\begin{array}{r}11.50 \\
(3.53) \\
\end{array}$ & $\begin{array}{c}9.11 \\
(3.18) \\
\end{array}$ & \begin{tabular}{|c|}
2.39 \\
$(1.84)$ \\
\end{tabular} & $\begin{array}{l}3.165 \\
(2.04) \\
\end{array}$ & $\begin{array}{c}7.67 \\
(2.94) \\
\end{array}$ & $\begin{array}{r}8.00 \\
(2.98) \\
\end{array}$ & $\begin{array}{r}1.89 \\
(1.69) \\
\end{array}$ & $\begin{array}{c}3.50 \\
(2.12) \\
\end{array}$ & $\begin{array}{c}5.39 \\
(2.53) \\
\end{array}$ & $\begin{array}{c}3.50 \\
(2.12) \\
\end{array}$ & 4.95 ef \\
\hline $\begin{array}{l}\text { 2. Imidacloprid } 60 \\
\text { FS }\end{array}$ & $\begin{array}{l}\mathrm{ml} / \mathrm{Kg} \\
\text { seeds }\end{array}$ & $\begin{array}{r}2.5 \\
(1.86) \\
\end{array}$ & $\begin{array}{c}3.67 \\
(2.15) \\
\end{array}$ & $\begin{array}{r}8.88 \\
(3.14) \\
\end{array}$ & $\begin{array}{c}7.28 \\
(2.88) \\
\end{array}$ & $\begin{array}{c}1.89 \\
(1.69) \\
\end{array}$ & $\begin{array}{c}2.16 \\
(1.78) \\
\end{array}$ & $\begin{array}{c}4.55 \\
(2.35) \\
\end{array}$ & $\begin{array}{r}5.00 \\
(2.43) \\
\end{array}$ & $\begin{array}{c}0.84 \\
(1.35) \\
\end{array}$ & $\begin{array}{c}1.83 \\
(1.67) \\
\end{array}$ & $\begin{array}{c}3.83 \\
(2.20) \\
\end{array}$ & $\begin{array}{c}1.95 \\
(1.72) \\
\end{array}$ & $7.32 \mathrm{~b}$ \\
\hline $\begin{array}{l}\text { 3. Imidacloprid } 60 \\
\text { FS }\end{array}$ & $\begin{array}{l}15 \mathrm{ml} / \mathrm{Kg} \\
\text { of seeds }\end{array}$ & $\begin{array}{r}2.22 \\
(1.79) \\
\end{array}$ & $\begin{array}{c}3.34 \\
(2.07) \\
\end{array}$ & $\begin{array}{c}8.17 \\
(3.03) \\
\end{array}$ & $\begin{array}{c}6.56 \\
(2.75) \\
\end{array}$ & $\begin{array}{c}1.61 \\
(1.60) \\
\end{array}$ & $\begin{array}{c}2.00 \\
(1.73) \\
\end{array}$ & \begin{tabular}{|c|}
3.89 \\
$(2.20)$ \\
\end{tabular} & $\begin{array}{r}4.61 \\
(2.34) \\
\end{array}$ & $\begin{array}{c}0.39 \\
(1.18) \\
\end{array}$ & $\begin{array}{c}1.34 \\
(1.52) \\
\end{array}$ & $\begin{array}{c}3.28 \\
(2.07) \\
\end{array}$ & $\begin{array}{c}1.73 \\
(1.65) \\
\end{array}$ & $8.68 \mathrm{a}$ \\
\hline $\begin{array}{l}\text { 4. Imidacloprid } 17.8 \\
\text { SL }\end{array}$ & $\begin{array}{l}0.3 \mathrm{ml} / \\
\mathrm{Lt}\end{array}$ & $\begin{array}{r}13.23 \\
(3.77) \\
\end{array}$ & $\begin{array}{c}4.73 \\
(2.39) \\
\end{array}$ & $\begin{array}{r}9.87 \\
(3.30) \\
\end{array}$ & $\begin{array}{c}6.45 \\
(2.72) \\
\end{array}$ & \begin{tabular}{|l|}
4.615 \\
$(2.37)$ \\
\end{tabular} & $\begin{array}{c}1.84 \\
(1.68) \\
\end{array}$ & $\begin{array}{c}5.17 \\
(2.48) \\
\end{array}$ & $\begin{array}{r}4.45 \\
(2.33) \\
\end{array}$ & $\begin{array}{c}4.67 \\
(2.38) \\
\end{array}$ & $\begin{array}{c}2.45 \\
(1.84) \\
\end{array}$ & $\begin{array}{c}3.72 \\
(2.17) \\
\end{array}$ & $\begin{array}{c}1.67 \\
(1.63) \\
\end{array}$ & $6.91 \mathrm{bc}$ \\
\hline $\begin{array}{l}\text { 5. Neem oil (1500 } \\
\text { ppm) }\end{array}$ & & $\begin{array}{r}13.39 \\
(3.79) \\
\end{array}$ & $\begin{array}{r}6.67 \\
(2.77) \\
\end{array}$ & $\begin{array}{r}11.84 \\
(3.58) \\
\end{array}$ & $\begin{array}{c}8.00 \\
(3.00) \\
\end{array}$ & \begin{tabular}{|c}
4.95 \\
$(2.43)$ \\
\end{tabular} & $\begin{array}{c}2.89 \\
(1.97) \\
\end{array}$ & \begin{tabular}{|c}
6.33 \\
$(2.69)$ \\
\end{tabular} & $\begin{array}{r}6.00 \\
(2.65) \\
\end{array}$ & $\begin{array}{c}4.45 \\
(2.33) \\
\end{array}$ & $\begin{array}{c}3.06 \\
(2.01) \\
\end{array}$ & $\begin{array}{c}5.67 \\
(2.58) \\
\end{array}$ & $\begin{array}{c}3.22 \\
(2.05) \\
\end{array}$ & $5.37 \mathrm{de}$ \\
\hline & $5 \%$ & $\begin{array}{r}12.89 \\
(3.72) \\
\end{array}$ & $\begin{array}{c}7.89 \\
(2.98) \\
\end{array}$ & $\begin{array}{r}14.50 \\
(3.93) \\
\end{array}$ & $\begin{array}{r}10.00 \\
(3.32) \\
\end{array}$ & $\begin{array}{c}5.05 \\
(2.46) \\
\end{array}$ & $\begin{array}{c}4.95 \\
(2.43) \\
\end{array}$ & $\begin{array}{c}8.5 \\
(3.08) \\
\end{array}$ & $\begin{array}{r}6.95 \\
(2.79) \\
\end{array}$ & $\begin{array}{c}5.11 \\
(2.47) \\
\end{array}$ & $\begin{array}{c}5.28 \\
(2.49) \\
\end{array}$ & $\begin{array}{c}6.84 \\
(2.79) \\
\end{array}$ & $\begin{array}{c}4.11 \\
(2.24) \\
\end{array}$ & $3.75 \mathrm{fg}$ \\
\hline $30 \mathrm{EC}$ & $\begin{array}{l}1.7 \mathrm{ml} / \\
\mathrm{Lt}\end{array}$ & $\begin{array}{r}13.17 \\
(3.76) \\
\end{array}$ & $\begin{array}{c}5.45 \\
(2.51) \\
\end{array}$ & $\begin{array}{l}10.00 \\
(3.31) \\
\end{array}$ & $\begin{array}{c}7.23 \\
(2.86) \\
\end{array}$ & $\begin{array}{c}5.11 \\
(2.47) \\
\end{array}$ & $\begin{array}{c}2.44 \\
(1.83) \\
\end{array}$ & \begin{tabular}{|c}
5.49 \\
$(2.54)$ \\
\end{tabular} & $\begin{array}{r}4.72 \\
(2.38) \\
\end{array}$ & $\begin{array}{c}4.50 \\
(2.34) \\
\end{array}$ & $\begin{array}{c}2.78 \\
(1.93) \\
\end{array}$ & $\begin{array}{c}5.00 \\
(2.44) \\
\end{array}$ & $\begin{array}{c}2.56 \\
(1.87) \\
\end{array}$ & $5.93 \mathrm{~cd}$ \\
\hline \multirow[t]{4}{*}{ 8. Control } & - & $\begin{array}{r}13.22 \\
(3.75) \\
\end{array}$ & $\begin{array}{l}22.22 \\
(4.80) \\
\end{array}$ & $\begin{array}{l}26.22 \\
(5.20) \\
\end{array}$ & $\begin{array}{l}21.17 \\
(4.68) \\
\end{array}$ & $\begin{array}{c}4.39 \\
(2.30) \\
\end{array}$ & $\begin{array}{c}9.15 \\
(3.16) \\
\end{array}$ & \begin{tabular}{|l}
14.50 \\
$(3.93)$ \\
\end{tabular} & $\begin{array}{r}11.50 \\
(3.53) \\
\end{array}$ & $\begin{array}{c}4.45 \\
(2.32) \\
\end{array}$ & $\begin{array}{c}8.34 \\
(3.05) \\
\end{array}$ & $\begin{array}{l}10.72 \\
(3.40) \\
\end{array}$ & $\begin{array}{c}6.78 \\
(2.77) \\
\end{array}$ & $2.87 \mathrm{gh}$ \\
\hline & SEm \pm & 0.13 & 0.13 & 0.15 & 0.12 & 0.09 & 0.11 & 0.13 & 0.11 & 0.09 & 0.10 & 0.13 & 0.10 & 0.39 \\
\hline & CD(0.05) & 0.38 & 0.41 & 0.44 & 0.35 & 0.26 & 0.32 & 0.38 & 0.34 & 0.27 & 0.31 & 0.40 & 0.31 & 1.18 \\
\hline & $\mathrm{CV}(\%)$ & 7.08 & 8.24 & 6.97 & 6.30 & 7.04 & 8.91 & 7.90 & 7.31 & 7.76 & 8.51 & 8.95 & 8.91 & 11.80 \\
\hline
\end{tabular}


Table.4 Cost economics of different seed treatment chemicals and plant products tested against sucking pests on Blackgram

\begin{tabular}{|c|c|c|c|c|c|c|c|c|}
\hline Treatments & Dose & $\begin{array}{l}\text { Yield } \\
(\mathrm{q} / \mathrm{ha})\end{array}$ & $\begin{array}{l}\text { Common } \\
\text { cost of } \\
\text { cultivation }\end{array}$ & $\begin{array}{l}\text { Treatment } \\
\text { cost }\end{array}$ & \begin{tabular}{|l} 
Total \\
cost
\end{tabular} & \begin{tabular}{|l} 
Gross \\
returns
\end{tabular} & $\begin{array}{l}\begin{array}{l}\text { Treatment } \\
\text { benefit }\end{array} \\
\end{array}$ & $\mathrm{B}: \mathrm{C}$ ratio \\
\hline 1. Imidacloprid $60 \mathrm{FS}$ & $\begin{array}{l}5 \mathrm{ml} / \mathrm{Kg} \\
\text { of seeds }\end{array}$ & 4.95 & 19776 & 320 & 20096 & 39600 & 16640 & 1.97 \\
\hline 2. Imidacloprid $60 \mathrm{FS}$ & $\begin{array}{l}10 \mathrm{ml} / \mathrm{Kg} \\
\text { of seeds }\end{array}$ & 7.32 & 19776 & 620 & 20396 & 58560 & 35600 & 2.87 \\
\hline 3. Imidacloprid $60 \mathrm{FS}$ & $\begin{array}{l}15 \mathrm{ml} / \mathrm{Kg} \\
\text { of seeds }\end{array}$ & 8.68 & 19776 & 920 & 20696 & 69440 & 46480 & 3.36 \\
\hline 4. Imidacloprid $17.8 \mathrm{SL}$ & $0.3 \mathrm{ml} / \mathrm{lt}$ & 6.91 & 19776 & 1000 & 20776 & 55280 & 32320 & 2.66 \\
\hline 5. Neem oil (1500 ppm) & $5 \mathrm{ml} / \mathrm{lt}$ & 5.37 & 19776 & 1400 & 21176 & 42960 & 20000 & 2.03 \\
\hline 6. NSKE & $5 \%$ & 3.75 & 19776 & 875 & 20651 & 30000 & 7040 & 1.45 \\
\hline 7. Dimethoate $30 \mathrm{EC}$ & $1.7 \mathrm{ml} / \mathrm{lt}$ & 5.93 & 19776 & 783 & 20559 & 47440 & 24480 & 2.31 \\
\hline 8. Control & 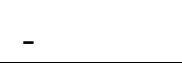 & 2.87 & 19776 & 0 & 19776 & 22960 & 0 & 1.16 \\
\hline
\end{tabular}

Imidacloprid 60 FS - Rs. 4000/Lt, Imidacloprid 17.8 SL - Rs. 2840/Lt, Neem oil - Rs. 400 / Lt, Neem seed kernel - Rs. 15/Kg

Dimethoate 30 EC - Rs. 375/Lt, Blackgram - Rs 8000/ Qtl., Labour cost - Rs 341.93/Lb/Day 
The effectiveness of seed dressing/ treatment chemicals against leafhopper was more or less similar to that of the aphids and thrips. Seed treatment with Imidacloprid 60 FS showed lowest leafhopper population which ranged from 0.56 to 1.89 Leafhopper/ top three leaves than other foliar spray treatments which showed 3.89 to 4.78 Leafhopper/ top three leaves at 15 days after sowing.

At 30 days after sowing lowest Leafhopper population of 1.67 and 2.33 Leafhopper / top three leaves was recorded in the plots treated with Imidacloprid 60 FS @ $15 \mathrm{ml} / \mathrm{Kg}$ of seeds and $10 \mathrm{ml} / \mathrm{kg}$ of seeds respectively.

These two treatments were followed by Imidacloprid $17.8 \mathrm{SL} @ 0.3 \mathrm{ml} / \mathrm{Lt}$ of water with 3.00 Leafhopper/top three leaves, Dimethoate 30 EC @ $1.7 \mathrm{ml} / / \mathrm{Lt}$ of water and Neem oil (1500 ppm) @ 5ml/Lt of water with 3.11 Leafhopper /top three leaves, Imidacloprid 60 FS @ $5 \mathrm{ml} / \mathrm{Kg}$ of seeds with 3.89 Leafhopper/top three leaves and NSKE $5 \%$ with 4.22 Leafhopper /top three leaves. The highest population of 7.56 Leafhopper /top three leaves was noticed in untreated control.

At 45 days after sowing Leafhopper population started to build up in all the treatment which ranged from 3.11 to 7.11 Leafhopper/top three leaves.

However, highest Leafhopper population was recorded in untreated control with 10.00 Leafhopper/ top three leaves. The same trend was followed at 60 days after sowing with decreasing in Leafhopper population in all treatments.

During 2015-16 (Table 2) same trend was followed with respect the aphid population observed on top three leaves per plant in each treatment at $15 \mathrm{DAS}, 30 \mathrm{DAS}, 45 \mathrm{DAS}$ and 60 DAS.

\section{Yield}

The highest average grain yield of 2014-15 and 2015-16 was recorded in Imidacloprid 60 FS@15 ml/ Kg of seeds (8.68 q/ha) and Imidacloprid 60 FS @10 ml/kg of seeds (7.32 q/ha) (Table 3). The treatment Imidacloprid 17.8 SL@0.3 ml/ Lt of water, Dimethoate 30 EC@1.7 ml// Lt of water, Neem oil (1500 ppm)@5ml/Lt of water, seed treatment with Imidacloprid 60 FS @ $5 \mathrm{ml} / \mathrm{Kg}$ of seeds and NSKE 5\% recorded $6.91 \mathrm{q} / \mathrm{ha}, 5.93 \mathrm{q} / \mathrm{ha}, 5.37$ $\mathrm{q} / \mathrm{ha}, \quad 4.95 \mathrm{q} / \mathrm{ha}, \quad 3.75 \mathrm{q} / \mathrm{ha}$ respectively. Untreated control recorded lowest grain yield of $2.87 \mathrm{q} / \mathrm{ha}$.

\section{B: C ratio}

Imidacloprid 60 FS @ $15 \mathrm{ml} / \mathrm{Kg}$ of seeds and Imidacloprid 60 FS @ $10 \mathrm{ml} / \mathrm{kg}$ of seeds (Table 4) recorded highest B: C ratio of 3.36 and 2.87. The treatment Imidacloprid 17.8 SL $@ 0.3 \mathrm{ml} / \mathrm{Lt}$ of water, Dimethoate $30 \mathrm{EC}$ @ $1.7 \mathrm{ml} / / \mathrm{Lt}$ of water, Neem oil (1500 ppm) @ 5ml/Lt of water, seed treatment with Imidacloprid 60 FS @ $5 \mathrm{ml} / \mathrm{Kg}$ of seeds and NSKE 5\% recorded 2.66, 2.31, 2.03, 1.97, $1.45 \mathrm{~B}: \mathrm{C}$ ratio respectively. Untreated control recorded lowest $\mathrm{B}$ : $\mathrm{C}$ ratio of 1.16 .

\section{Effect on natural enemies}

The activity of the natural enemies was reduced in the treated plots compared to the untreated control as the population of the sucking pests was very low in treated plots.

\section{Phytotoxicity}

There was no Phytotoxicity symptoms were noticed on the crop after imposing the treatments. Further, it should be kept in the mind that, we need to sow the seeds treated with Imidacloprid 60 FS when we have the optimum moisture/ enough moisture in the soil. 
As this is the first study by using seed treatment chemicals to manage the sucking pests of blackgram, no reviews related to this topic is available in blackgram. However, the other reviews are available by using Imidacloprid with different formulation as a seed treatment to manage early stage sucking pests in blackgram, cotton and bhendi; hence those reviews are used to compare the present results.

From the present study, the result revealed that Imidacloprid 60 FS @ 15 ml/ Kg of seeds and Imidacloprid 60 FS @ $10 \mathrm{ml} / \mathrm{kg}$ of seeds effectively reduced the sucking pest population in the blackgram field. The present results are in accordance with the results of Soundarajan and Chitra (2011) who reported that seed treatment by Imidacloprid recorded the lowest incidence of the sucking pests in blackgram. Further, these results were almost similar to the findings of Mote et al., (1995) and Patil et al., (2003) who observed that Imidacloprid as seed treating chemical reduced sucking pest population below the economic threshold level up to 40 days after sowing in cotton and 61 days after germination (Dandale et al., 2001: Murugan et al., 2003) in cotton. Sreenivas and Nargund, 2006 reported that Imidacloprid $@ 5 \mathrm{~g} / \mathrm{kg}$ of seeds as seed dressing chemical will protect the bhendi crop up to 50 days from sucking pests. Murugan and Kavitha (2009) reported that Imidacloprid recorded the least mean population of leafhoppers in cotton. Imidacloprid (Gaucho 70 WS) effectively reduced population of aphids, whiteflies and thrips in cotton Hossain et al., 2013. Harish kumar et al.,2013 reported that Imidacloprid 600FS when applied as seed treatment was most effective in controlling the sucking pests up to four week of seed germination in soybean. The seed treatment with Imidacloprid 60 FS @ 5 ml/kg of seeds was less effective compared to other two treatments and this result was in accordance with Iqbal et al., (2013) who reported that seed treatment with Imidacloprid 60 FS @5 $\mathrm{g} / \mathrm{kg}$ of seeds along with detergent $2 \mathrm{~g} / \mathrm{l}$ of water proved less effective in managing the sucking pests greengram.

Seed treatment with Imidacloprid60 FS @ 10 $\mathrm{ml} / \mathrm{kg}$ seeds sown when there is sufficient moisture soil was most effective in controlling the sucking pests of blackgram starting from sowing/ germination up to 40-45 days old crop with highest grain yield and proved cost effective.

\section{References}

Dandale, H. G., A. Y. Thakare, S. N. Tikar, N. G. V. Rao and S. A. Nimblakar. 2001. Effect of seed treatment on sucking pests of cotton and yield of seed cotton. Pestology 25: 20-23.

Dhawan, A. K. and G. S. Simwat. 2002. Field evaluation of thiamethoxam for control of cotton jassid Amrasca biguttula (Ishida) on upland cotton. Pestology 26: 15-19.

Harish kumar Netam, Rajeev gupta and Shivam soni. 2013. Bioefficacy of insecticides as seed treatment against early sucking pests of soybean crop. International Journal of Science and Research. 2 (1):688-690.

Hossain, S. M.A., Baque, M. A., and Amin, M.R. 2013. Comparative effectiveness of seed treating and foliar insecticides against sucking pests of cotton and impact on their natural enemies. Bangladesh Journal of Agriculture research. 38 (1): 61-70.

Jamshaid Iqbal, Muhammad Nadeem, Muhammad Saddique Assi, Malik Muhammad Fiaz and Muhammad Waqas Ul Hassan.2013. Gomal University Journal of Research. 29(1):31-37.

Magalhaes, L. C., T. E. Hunt and B. D. 
Siegfried. 2009. Efficacy of neonicotinoid seed treatments to reduce soybean aphid populations under field and controlled conditions in Nebraska. J. Econ. Entomol. 102: 187-195.

Maienfisch, P., M. Angst, F. Brandl, W. Fischer, D. Hofer and H. Kayser. 2001. Chemistry and biology of thiamethoxam: a second generation neonicotinoid. Pest Manag. Sci. 57: 906-913.

Mote, U. N., R. V. Datkile and G. R. Loage. 1995 Efficacy of Imidacloprid as seed treatment against initial sucking pests of cotton. Pestology. 19: 5-8.

Murugan, M. N. Sathiah, N. Dhandapani, R. J. Rabindra and S. Mohan. 2003. Laboratory assays on the role of Indian transgenic Bt cotton in the management of Helicoverpa armigera (Hubner) (Noctuidae: Lepidoptera). Indian J. Plant protection 31: 1-5.

Murugan, N. and Kavitha, A. 2009. Seed treatment with Pseudomonas fluorescens, plant products and synthetic insecticides against the leafhopper, Amrasca devastans (Distant) in cotton. Journal of Biopesticides, 2 (1): 22-25.

Nault, B. A., A. G. Taylor, M. Urwiler, T. Rabaey and W. D. Hutchison. 2004. Neonicotiniod seed treatments for managing potato leafhopper infestations in snap bean. Crop Protection 23: 147154.

Patil, B. C., S. B. Patil, S. S. Vdikeri and B. M. Khadi. 2003. Effect of Imidacloprid seed treatment on growth, yield, seedling vigor and biophysical parameters in cotton (Gossypium spp) genotypes. In: Proc. World Cotton Res. Conf. 3, Cape Town, South Africa, 9-13 march 2003.

Soundarajan, R. P. and Chitra, N. 2011. Effect of bioinoculants on sucking pests and pod borer complex in urdbean. Journal of Biopesticides.4(1): 7-11

Sreenivas, A. G. and Nargund, V. B. 2006. Management of sucking insect pests of Bhendi through seed dressing chemicals. Karnataka Journal of Agricultural Sciences, 19(2): 307-311.

Taylor, A. G., C. J. Eckenrode and R. W. Straub. 2001. Seed coating technologies and treatments for onions: challenges and progress. Hort. Sci. 36: 199-205.

Udikeri, S. S., S. B. Patil, L. K. Naik, V. Rachappa, F. Nimbal and G. S. Guruprasad. 2007. Poncho 600 FS - a new seed dressing formulation for sucking pest management in cotton. Karnataka J. Agric. Sci. 20: 51-53.

Vadodaria, M. P., U. G. Patel, C. J. Patel, R. B. Patel and I. M. Maisuria. 2001. Thiamethoxam (Cruiser) 70 WS: a new seed dresser against sucking pests of cotton. Pestology 25: 13-18.

Zhang, L., S. M. Greenberg, Y. Zhang and T. Liu. 2011. Effectiveness of thiamethoxam and imidacloprid seed treatments against Bemisia tabaci (Hemiptera: Aleyrodidae) on cotton. Pest Manag. Sci. 67: 226-232.

\section{How to cite this article:}

Shobharani, M., Sidramappa and Sunilkumar, N.M. 2017. Management of Sucking Pests of Blackgram Using Seed Treatment Chemicals. Int.J.Curr.Microbiol.App.Sci. 6(12): 3374-3383. doi: https://doi.org/10.20546/ijcmas.2017.612.393 